\title{
Simultaneous-Perturbation-Stochastic-Approximation Algorithm for Parachute Parameter Estimation
}

\author{
Govindarajan Kothandaraman* and Mario A. Rotea ${ }^{\dagger}$ \\ Purdue University, West Lafayette, Indiana 47907-1282
}

\begin{abstract}
This paper presents an algorithm to estimate unknown parameters of parachute models from flight-test data. The algorithm is based on the simultaneous-perturbation-stochastic-approximation method to minimize the prediction error (difference between model output and test data). The algorithm is simple to code and requires only the model output. Analytical gradients are not necessary. The algorithm is used to estimate aerodynamic and apparent mass coefficients for an existing parachute model.
\end{abstract}

\section{Nomenclature}

\section{$A$}

$B, C, D$

$C_{d}$

$C_{m}$

$c_{\text {ref }}$

$F_{x}, F_{y}, F_{z}$

$H$

$I_{x x}, I_{y y}, I_{z z}$

$M_{x}, M_{y}, M_{z}$

$m$

$p$

$q$

$r$

$S_{\text {ref }}$

$U$

$u, v, w$

V

$X, Y, Z$

$x, y, z$

$\alpha$

$\alpha_{T}$

$\beta$

$\theta$

$\vartheta$

$\rho$

$\phi$

$\psi$

$=$
$=$
$=$
$=$
$=$
$=$
$=$
$=$
$=$
$=$
$=$

apparent mass tensor

points on parachute

drag coefficient

moment coefficient

reference length

quaternions

cost function

mass

= roll rate

$=$ pitch rate

$=$ yaw rate

$=$ reference area

- inertial coordinates

$=$ body coordinates

$=$ angle of attack

$=$ total angle of attack

$=$ angle of sideslip

$=$ pitch angle

$=$ unknown parameters

$=$ roll angle

= yaw angle $e_{0}, e_{1}, e_{2}, e_{3}$

force along $x, y$, and $z$ directions

moment of inertia about $x, y$, and $z$ axes

moment about $x, y$, and $z$ axes

$=$ velocity along $X$ direction

$=$ velocity along the $x, y$, and $z$ axes

$=$ velocity, velocity along $Y$ direction

$=$ density of air

\section{Introduction}

T HIS paper describes a simple algorithm for parameter estimation that can be used with nonlinear dynamic parachute models. Model parameters are determined by minimizing the prediction error obtained by comparing model output with test data. Minimization is accomplished using the simultaneous-perturbationstochastic-approximation (SPSA) algorithm developed by Spall. ${ }^{1,2}$

The SPSA algorithm is an iterative method for optimization, with randomized search direction, that requires at most three function

\footnotetext{
Presented as Paper 2003-2118 at the AIAA 17th Aerodynamic Decelerator Systems Technology Conference and Seminar, Monterey, CA, 19-22 May 2003; received 22 June 2004; revision received 15 September 2004; accepted for publication 24 September 2004. Copyright (C) 2004 by the American Institute of Aeronautics and Astronautics, Inc. All rights reserved. Copies of this paper may be made for personal or internal use, on condition that the copier pay the $\$ 10.00$ per-copy fee to the Copyright Clearance Center, Inc., 222 Rosewood Drive, Danvers, MA 01923; include the code 0021-8669/05 $\$ 10.00$ in correspondence with the CCC.

*Graduate Student, School of Aeronautics and Astronautics.

†Professor, School of Aeronautics and Astronautics; rotea@purdue.edu. Member AIAA.
}

(model) evaluations at each iteration. Hence, execution time per iteration does not increase with the number of parameters. The method can handle nonlinear dynamic models, nonequilibrium transient test conditions, and data obtained in closed loop. For this reason, this method is suitable for the estimation of parameters in guided parachute models.

The present paper has three main sections. The first section describes the model whose parameters are to be determined. The model is for a G-12 parachute, and it was developed at the Naval Postgraduate School (NPS). ${ }^{3,4}$ The second section explains the basic parameter estimation approach. This section includes a simple description of the SPSA algorithm. In the third section we give the numerical results corresponding to the determination of three aerodynamic coefficients, four apparent mass coefficients, and the initial states for the G-12 parachute model. Conclusions and recommendations for further work are the end of the paper.

\section{Parachute Model}

A six-degrees-of-freedom model of a fully deployed G-12 parachute was developed at NPS. ${ }^{3,4}$ Figure 1 gives a schematic of the G-12 parachute. This model assumes the following:

1) The parachute canopy and payload form one rigid system.

2) The aerodynamic forces and moments of the payload are negligible.

3) The aerodynamic forces act at the center of pressure of the canopy, which is nothing but the centroid of the air in the canopy.

4) The G-12 system is symmetrical about the axis joining the canopy centroid to the payload centroid.

5) The parachute is fully deployed.

\section{Equations of Motion}

Let $m$ be the total mass of the parachute system. Let $u, v$, and $w$ be the components of the ground velocity of the parachute in the body coordinate system (see Fig. 1). Let $p, q$, and $r$ be the components of the angular velocity of the parachute expressed in body coordinates. Then, the equations of motion of the parachute are as follows. ${ }^{3}$

$$
F_{x}=\left(m+A_{11}\right)(\dot{u}-v r)+\left(m+A_{33}\right) q w+\left(J 1+A_{15}\right)(\dot{q}+r p)
$$

$$
F_{y}=\left(m+A_{11}\right)(\dot{v}+u r)-\left(m+A_{33}\right) p w-\left(J 1+A_{15}\right)(\dot{p}-q r)
$$

$$
F_{z}=\left(m+A_{33}\right) \dot{w}-\left(m+A_{11}\right)(u q-v p)-\left(J 1+A_{15}\right)\left(p^{2}+q^{2}\right)
$$

$$
\begin{aligned}
M_{x}= & \left(I_{x x}+A_{55}\right) \dot{p}-\left(J 1+A_{15}\right)(\dot{v}-p w+u r) \\
& -\left(I_{y y}+A_{55}-I_{z z}\right) q r+\left(A_{33}-A_{11}\right) v w
\end{aligned}
$$




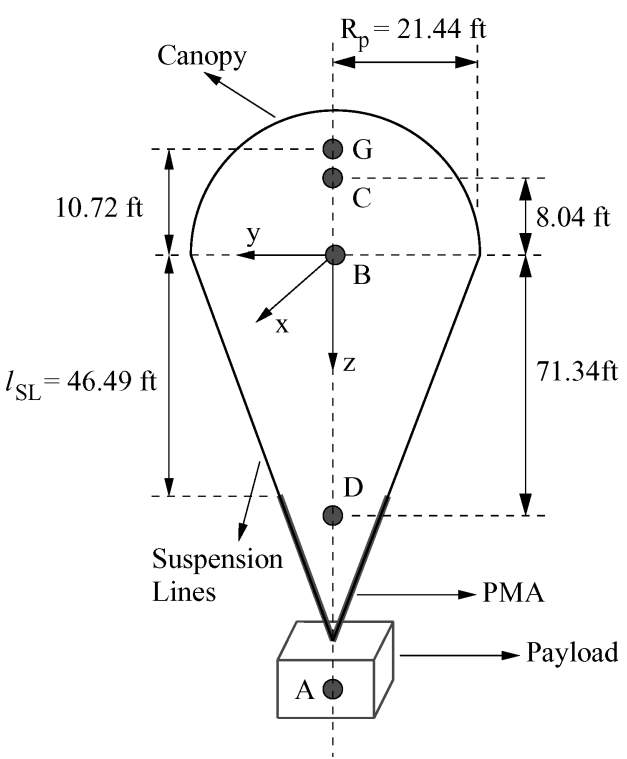

Fig. 1 G-12 parachute dimensions (not to scale).

$$
\begin{aligned}
M_{y}= & \left(I_{y y}+A_{55}\right) \dot{q}+\left(J 1+A_{15}\right)(\dot{u}+q w-r v) \\
& +\left(I_{x x}+A_{55}-I_{z z}\right) p r-\left(A_{33}-A_{11}\right) u w \\
M_{z}= & I_{z z} \dot{r}+\left(I_{y y}-I_{x x}\right) p q
\end{aligned}
$$

where

$$
\begin{gathered}
J 1=\sum_{i=1}^{n} m_{i} z_{i} \\
\sum_{i=1}^{n} m_{i}=m
\end{gathered}
$$

and $z_{i}$ is the location of the mass center of each of the component masses $m_{i}$ of the parachute system. The terms $A_{11}, A_{33}, A_{15}$, and $A_{55}$ are the "apparent mass" terms and are added to account for the acceleration of the fluid around the body. Expressions for the apparent mass terms were taken from Doherr and Saliaris ${ }^{5}$ and are given by

$$
\begin{gathered}
A_{11}=(\pi / 3) \rho R_{p}^{3} \\
A_{15}=0.2 A_{11} \sqrt{R_{p}^{2}+\ell_{\mathrm{SL}}^{2}} \\
A_{33}=2 A_{11} \\
A_{55}=0.192 R_{p}^{2} A_{11}
\end{gathered}
$$

where $R_{p}$ is the radius of the inflated canopy and $\ell_{\mathrm{SL}}$ is the length of the suspension lines projected onto the symmetry axis.

The following kinematic equations are used to determine the attitude of the parachute:

$$
\begin{gathered}
\dot{\phi}=p+q \sin \phi \tan \theta+r \cos \phi \tan \theta \\
\dot{\theta}=q \cos \phi-r \sin \phi \\
\dot{\psi}=q \sec \theta \sin \phi+r \sec \theta \cos \phi
\end{gathered}
$$

Finally, the inertial position of the origin of the body coordinates is obtained by integrating Eq. (7):

$\dot{X}=u \cos \psi \cos \theta+v(\cos \psi \sin \theta \sin \phi-\sin \psi \cos \phi)$

$$
+w(\cos \psi \sin \theta \cos \phi+\sin \psi \sin \phi)
$$

$$
\begin{aligned}
\dot{Y}= & u \sin \psi \cos \theta+v(\sin \psi \sin \theta \sin \phi+\cos \psi \cos \phi) \\
& +w(\sin \psi \sin \theta \cos \phi-\cos \psi \sin \phi) \\
\dot{Z}= & -u \sin \theta+v \cos \theta \sin \phi+w \cos \theta \cos \phi
\end{aligned}
$$

Thus the 12 states can be determined using Eqs. (1) and (2) and (6) and (7). See the thesis of Junge ${ }^{3}$ for further details.

\section{Forces and Moments}

Before the expressions for the forces and moments are given, certain definitions will be useful. The velocity of the parachute relative to the air is denoted by $\boldsymbol{V}_{\text {air }}$ and has components $u_{\text {air }}, v_{\text {air }}$, and $w_{\text {air }}$ in the body coordinates given by

$$
\left\{\begin{array}{c}
u_{\text {air }} \\
v_{\text {air }} \\
w_{\text {air }}
\end{array}\right\}=\left\{\begin{array}{c}
u \\
v \\
w
\end{array}\right\}-\left\{\begin{array}{c}
u_{\text {wind }} \\
v_{\text {wind }} \\
w_{\text {wind }}
\end{array}\right\}
$$

where $u_{\text {wind }}, v_{\text {wind }}$, and $w_{\text {wind }}$ are the components of the wind velocity in body coordinates.

The various flight angles used in the determination of force and moment coefficients are as follows. The total angle of attack is defined by

$$
\alpha_{T}=\arccos \left(\frac{w_{\text {air }}}{\sqrt{u_{\text {air }}^{2}+v_{\text {air }}^{2}+w_{\text {air }}^{2}}}\right)
$$

The angle of attack is given by

$$
\alpha=\arctan \left(\frac{u_{\text {air }}}{w_{\text {air }}}\right)
$$

The sideslip angle is given by

$$
\beta=\arctan \left(\frac{v_{\text {air }}}{\sqrt{u_{\text {air }}^{2}+w_{\text {air }}^{2}}}\right)
$$

The net force on the G-12 parachute is a result of the aerodynamic force and gravity. The aerodynamic force $\boldsymbol{F}^{\text {aero }}$ is assumed to act at the centroid of the canopy $C$ and has components $F_{x}^{\text {aero }}, F_{y}^{\text {aero }}$, and $F_{z}^{\text {aero }}$ along the body axes. These components are given by the following relation:

$$
\left\{\begin{array}{l}
F_{x}^{\text {aero }} \\
F_{y}^{\text {aero }} \\
F_{z}^{\text {aero }}
\end{array}\right\}=\frac{1}{2} \rho \sqrt{u_{\text {air }}^{2}+v_{\text {air }}^{2}+w_{\text {air }}^{2}} S_{\text {ref }} C_{d}\left(\alpha_{T}\right)\left\{\begin{array}{c}
u_{\text {air }} \\
v_{\text {air }} \\
w_{\text {air }}
\end{array}\right\}
$$

The density of air $\rho$ is a function of the altitude, and a standard atmospheric model was used. ${ }^{6}$

The drag coefficient $C_{d}$ depends on the total angle of attack $\alpha_{T}$, and it is shown in Fig. 2. This curve was obtained from the computations of Mosseev. ${ }^{7}$

The gravitational force $\boldsymbol{F}^{\text {grav }}$ acts along the inertial $Z$ direction and has the components $F_{x}^{\text {grav }}, F_{y}^{\text {grav }}$, and $F_{z}^{\text {grav }}$ in body coordinates given by

$$
\left\{\begin{array}{l}
F_{x}^{\text {grav }} \\
F_{y}^{\text {grav }} \\
F_{z}^{\text {grav }}
\end{array}\right\}=\left\{\begin{array}{c}
-\sin \theta \\
\cos \theta \sin \phi \\
\cos \theta \cos \phi
\end{array}\right\} \mathrm{mg}
$$

The net force in the left-hand side of Eq. (1) is the sum of forces in Eqs. (12) and (13).

The moments on the parachute are caused by the forces described earlier as well as the aerodynamic moments. The aerodynamic moment $\boldsymbol{M}^{\text {aero }}$ has components $M_{x}^{\text {aero }}, M_{y}^{\text {aero }}$, and $M_{z}^{\text {aero }}$ in the body coordinates given by

$$
\left\{\begin{array}{l}
M_{x}^{\text {aero }} \\
M_{y}^{\text {aero }} \\
M_{z}^{\text {aero }}
\end{array}\right\}=\frac{1}{2} \rho\left(u_{\text {air }}^{2}+v_{\text {air }}^{2}+w_{\text {air }}^{2}\right) S_{\text {ref }} C_{\text {ref }}\left\{\begin{array}{c}
C_{m}(\beta) \\
C_{m}(\alpha) \\
0
\end{array}\right\}
$$




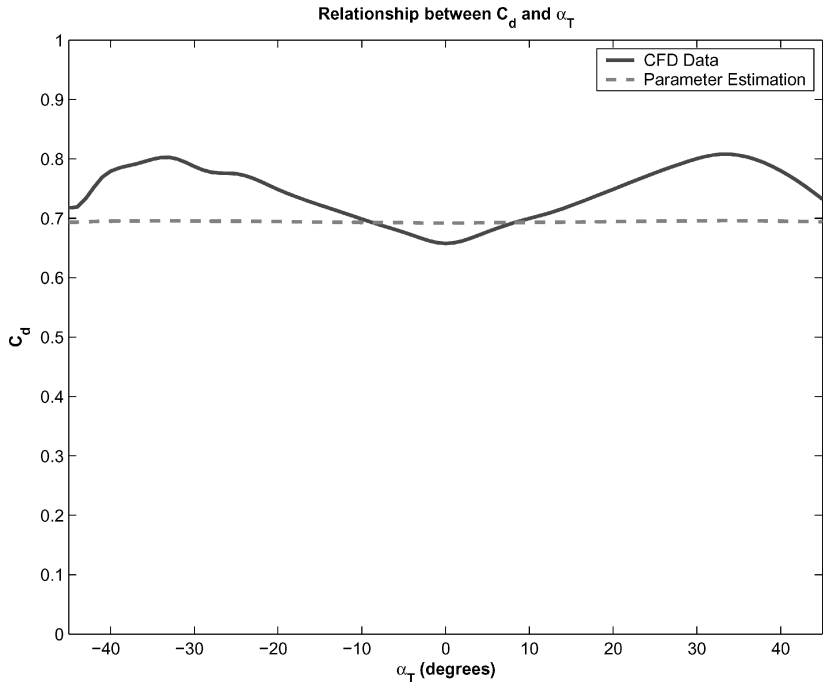

Fig. 2 Drag coefficient: - computational-fluid-dynamics (CFD) analysis ${ }^{7}$; and ---, parameter estimation result from fourth section.

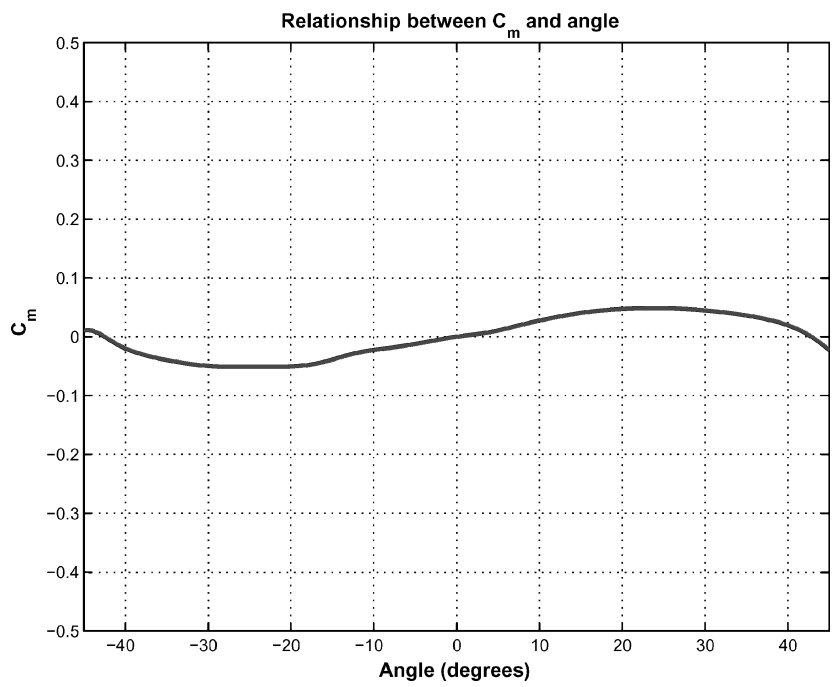

Fig. 3 Moment coefficient from CFD analysis. ${ }^{7}$

where the variation of $C_{m}$ with angle of attack $\alpha$ or sideslip $\beta$ is given in Fig. 3. This variation was also obtained from the computations of Mosseev. ${ }^{7}$

As mentioned earlier, the moment caused by the aerodynamic forces (assumed to act at the canopy centroid, point $C$ ) and weight (acting at the mass center, point $D$ ) also need to be accounted for in the final moment that is to be applied to the equations of motion. This moment, denoted $\boldsymbol{M}^{F}$, is given by

$$
\boldsymbol{M}^{F}=\boldsymbol{R}_{\mathrm{CB}} \times \boldsymbol{F}^{\text {aero }}+\boldsymbol{R}_{\mathrm{DB}} \times \boldsymbol{F}^{\text {grav }}
$$

where the moment arm $\boldsymbol{R}_{\mathrm{CB}}$ is the distance between the canopy centroid (point $C$ ) and the origin of the body frame (point $B$ ) and $\boldsymbol{R}_{\mathrm{DB}}$ is the distance between the center of mass (point $D$ ) of the system and the origin (see Fig. 1). For more details, the reader is referred to the thesis of Junge. ${ }^{3}$

The objective of this paper is to improve the parachute model using parameter estimation. In the next section, the $C_{d}$ and the $C_{m}$ curves will be modified, and the parameters that modify these curves will be estimated. Also, the apparent mass terms in Eq. (5) will be modified, and parameters modifying these terms will be estimated. Finally, the initial conditions of the states will also be estimated.

\section{Parameter Estimation}

Once the model structure and the test data are known, the next step is to estimate the parameters of the system. This is done by assuming an initial value of the parameters and then optimizing them so as to minimize the error between the measurements and the model predictions. In this work, a code using standard MATLAB ${ }^{\circledR}$ commands, implementing the SPSA algorithm ${ }^{1,8}$ for constrained optimization, was developed.

The model given in the preceding section was modified to reduce the model errors. The modifications are as follows. The aerodynamic force term is determined by Eq. (12), where the value of the drag coefficient is determined using a look-up table corresponding to Fig. 2. Let this function be denoted by $C_{d}^{*}(\cdot)$. Consider the following modification to the drag coefficient:

$$
C_{d}(\cdot)=\vartheta_{1}+\vartheta_{2} C_{d}^{*}(\cdot)
$$

where $\vartheta_{1}$ is an offset and $\vartheta_{2}$ is a scale factor, which need to be determined to reduce the model error.

Similarly, consider the moment term. The aerodynamic moment is given by Eq. (14), where the value of the moment coefficient is given by Fig. 3. Let this function be denoted by $C_{m}^{*}(\cdot)$. Consider the following modification to the moment coefficient:

$$
C_{m}(\cdot)=\vartheta_{3} C_{m}^{*}(\cdot)
$$

Note that the physics of the problem does not allow for a nonzero $C_{m}$ at zero angle of attack, which explains the absence of an offset term for the moment coefficient.

Scale factors $\vartheta_{4}, \vartheta_{5}, \vartheta_{6}$, and $\vartheta_{7}$ were incorporated in the apparent mass terms in Eq. (5) as well, which resulted in

$$
\begin{gathered}
A_{11}=\vartheta_{4}(\pi / 3) \rho R_{p}^{3} \\
A_{15}=0.2 \vartheta_{5} A_{11} \sqrt{R_{p}^{2}+\ell_{\mathrm{SL}}^{2}} \\
A_{33}=2 \vartheta_{6} A_{11} \\
A_{55}=0.192 \vartheta_{7} R_{p}^{2} A_{11}
\end{gathered}
$$

The initial conditions of the 12 states were also estimated to get a better fit of the data to the model. This gives an additional 12 unknowns, $\vartheta_{8}, \ldots, \vartheta_{19}$.

Thus the vector of unknowns $\vartheta$ is given by

$$
\vartheta=\{\underbrace{\vartheta_{1}, \vartheta_{2}, \vartheta_{3}}_{\text {Aerodynamic parameters }}, \underbrace{\vartheta_{4}, \ldots, \vartheta_{7}}_{\text {Apparent mass terms }}, \underbrace{\vartheta_{8}, \ldots, \vartheta_{19}}_{\text {Initial conditions }}\}^{T}
$$

\section{Method of Parameter Estimation}

The prediction error method (PEM) was used to estimate the parameters $\vartheta$ in Eq. (19). PEM estimates these parameters by minimizing the difference between the experimental data and the model output.

PEM works by minimizing a cost function. There are many cost functions that can be chosen. Let the measured output vector at time instant $k$ be denoted by $y(k)$, and let the predicted output vector at time $k$ using parameters $\vartheta$ be denoted by $\hat{y}(k ; \vartheta)$. Define the prediction error as

$$
e(k ; \vartheta)=y(k)-\hat{y}(k ; \vartheta)
$$

A common cost function is the quadratic function given by

$$
H(\vartheta)=\sum_{k=1}^{N} e^{T}(k ; \vartheta) P e(k ; \vartheta)
$$

where $N$ is the total number of time samples and $P$ is a weighting matrix. The matrix $P$ was chosen to be diagonal, and the values of the diagonal elements were chosen so that all the components of $e(k ; \vartheta)$ were normalized and nondimensionalized. This was achieved by 
choosing the diagonal elements to be the inverse of the square of the infinity norm of the measured outputs, that is,

$$
P(i, j)=\left\{\begin{array}{lll}
0, & \text { if } & i \neq j \\
\frac{1}{\left\|y_{i}\right\|_{\infty}^{2}}, & \text { if } & i=j
\end{array}\right.
$$

where $y_{i}$ is the $i$ th measurement vector. Once this value of $P$ is determined, it can be adjusted to give different weights to different output channels.

\section{Optimization}

A vector of optimal parameters $\hat{\vartheta}$ is obtained by solving the following problem:

$$
\begin{gathered}
\hat{\vartheta}=\arg \min _{\vartheta} H(\vartheta) \\
\text { subject to } \quad \vartheta^{\min } \leq \vartheta \leq \vartheta^{\max }
\end{gathered}
$$

where the inequality constraint is component-wise and the cost function $H(\vartheta)$ is given in Eq. (21).

Most optimization methods use the gradient and possibly the Hessian of the cost function $H$ to arrive at a local minimum. The accuracy and efficiency of these methods depends on how the gradient and/or the Hessian are obtained. In this work, we selected the SPSA method for optimization. ${ }^{1}$ This method is an iterative method that refines the optimization variables at each iteration using a numerically computed estimation for the gradient. In our application, SPSA is an attractive choice for the following reasons. Analytical gradients are not required, and only a fixed number of cost functions evaluations (three in our case) is required to estimate the gradient and update the optimization variables. The calculation of the estimated gradient is based on a randomized algorithm.

Spall $^{1,2}$ describes the SPSA algorithm, gives the theoretical and numerical properties of the algorithm, and proves its convergence in a stochastic sense. ${ }^{9}$ Sadegh ${ }^{8}$ and Wang and Spall ${ }^{10}$ describe how the SPSA can be used for constrained optimization as well. The rest of this section is devoted to a brief explanation of the SPSA algorithm, which according to Spall ${ }^{1}$ has the following property: "One properly chosen simultaneous random change in all the variables in a problem provides as much information for optimization as a full set of one-ata-time changes of each variable." It is this property that makes SPSA attractive in our application. Roughly, this property allows SPSA to estimate the gradient of the cost function $H$ with two function evaluations regardless of the number of optimization variables.

The basic SPSA algorithm we implemented is as follows:

1) Initialization and coefficient selection: Set counter index $k=1$. Pick initial guess for $\vartheta$ and nonnegative coefficients $a, c, A, \alpha$, and $\gamma$. Define the SPSA gain sequences $a_{k}=a /(A+k)^{\alpha}$ and $c_{k}=c / k^{\gamma}$.

2) Generation of the simultaneous perturbation vector: Let $\Delta_{k 1} \ldots \Delta_{k n}$ denote the $n$ scalar components of the random perturbation vector $\Delta_{k}$. Generate $\Delta_{k i}$ from a \pm 1 Bernoulli distribution with probability $\frac{1}{2}$ for each \pm 1 outcome.

3) Cost function evaluations: Obtain two measurements of the cost function $H(\cdot)$ based on the simultaneous perturbation around the current $\hat{\vartheta}_{k}$. That is, evaluate $H\left(\hat{\vartheta}_{k}+c_{k} \Delta_{k}\right)$ and $H\left(\hat{\vartheta}_{k}-c_{k} \Delta_{k}\right)$ with the $c_{k}$ and $\Delta_{k}$ from steps 1 and 2 .

4) Gradient approximation: Generate the simultaneous perturbation approximation to the unknown gradient $g\left(\hat{\vartheta}_{k}\right)$ as follows:

$$
\hat{g}_{k}\left(\hat{\vartheta}_{k}\right)=\frac{H\left(\hat{\vartheta}_{k}+c_{k} \Delta_{k}\right)-H\left(\hat{\vartheta}_{k}-c_{k} \Delta_{k}\right)}{2 c_{k}}\left[\begin{array}{c}
\Delta_{k 1}^{-1} \\
\Delta_{k 2}^{-1} \\
\vdots \\
\Delta_{k n}^{-1}
\end{array}\right]
$$

where $\Delta_{k i}$ is the $i$ th component of the vector $\Delta_{k}$.

5) Updating the parameter estimates: Use

$$
\hat{\vartheta}_{k+1}=\hat{\vartheta}_{k}-a_{k} \hat{g}_{k}\left(\hat{\vartheta}_{k}\right)
$$

to update $\hat{\vartheta}_{k}$ to a new value $\hat{\vartheta}_{k+1}$. If $\hat{\vartheta}_{k+1}$ falls outside the range of allowable values for $\vartheta$, then project the updated $\hat{\vartheta}_{k+1}$ to the nearest boundary value, and reassign this projected value as $\hat{\vartheta}_{k+1}$ (Ref. 10). Mathematically we have, for every $i=1, \ldots, n$, the following:

$$
\hat{\vartheta}_{k+1, i}=\left\{\begin{array}{lll}
\hat{\vartheta}_{k+1, i} & \text { if } & \vartheta_{i}^{\min } \leq \hat{\vartheta}_{k+1, i} \leq \vartheta_{i}^{\max } \\
\vartheta_{i}^{\min } & \text { if } & \hat{\vartheta}_{k+1, i}<\vartheta_{i}^{\min } \\
\vartheta_{i}^{\max } & \text { if } & \hat{\vartheta}_{k+1, i}>\vartheta_{i}^{\max }
\end{array}\right.
$$

If the basic update indicated in Eqs. (25) and (26) produces a higher value of the cost function than the previous one, then the update is blocked, and we set $\hat{\vartheta}_{k+1}=\hat{\vartheta}_{k}$.

6) Iteration or termination: Terminate the algorithm if there is little change in several iterates or if the maximum number of allowable iterations has been reached. Otherwise, return to step 2 with $k+1$ replacing $k$.

The choice of various parameters of the algorithm plays an important role in the convergence of the algorithm. Spall ${ }^{2}$ suggests that $\alpha=0.602$ and $\gamma=0.101$ are practically effective and theoretically valid choices. Hence these values were used in this paper as well. The value of $A$ is chosen to be about $10 \%$ of the maximum iterations allowed. The maximum number of iterations was chosen to be 100 , and hence $A$ was chosen to be 10 . Spall ${ }^{2}$ recommends that if the measurements are (almost) error free, $c$ can be chosen as a small positive number. In this case it was chosen to be $c=0.01$. Spall ${ }^{2}$ explains that the value of $a$ should be chosen such that the $a /(A+1)^{\alpha}$ times the magnitude of elements of $\hat{g}_{0}\left(\hat{\vartheta}_{0}\right)$ is approximately equal to the smallest of the desired change in magnitudes among the elements of $\vartheta$ in early iterations. For the problem at hand $a=1$ gave good results. This value of $a$ was chosen to increase the likelihood that the components of $\vartheta$ during the iterations remain within the allowed bounds, namely, $\vartheta_{\min }^{i}$ to $\vartheta_{\max }^{i}$. In case one or more of the components of $\hat{\vartheta}_{k}+c_{k} \Delta_{k}$ or $\hat{\vartheta}_{k}-c_{k} \Delta_{k}$ was outside the allowed limits, the corresponding perturbed parameter was projected to the nearest boundary to compute the estimate of the gradient.

The proposed algorithm uses three cost function computations in each iteration. Thus, the number of cost function evaluations per iteration does not depend on the number of variables, which makes this method very attractive for optimization problems with several variables or with computationally costly function evaluations. This is in contrast to the numerical computation of gradients by finite differences where the number of cost function evaluations increases linearly with the number of variables to be optimized.

\section{Results}

This section gives the results of parameter estimation where all 12 initial conditions and seven model parameters are determined [see Eq. (19)]. These parameters are estimated using SPSA. Forty cases were run to determine a simple statistics for the cost function $H$ and the estimated parameters. It is important to run many cases because the results can then be used, as will be seen later, in a statistical study to decide if the parameter estimation is successful. Essentially, the distribution of the cost function $H$ over all of the cases considered can be used to assess if the algorithm "converges," whereas the distributions of the estimated parameters can be used to determine if the parameters are actually "identifiable" from the given test data.

The initial guesses for the aerodynamic and apparent mass parameters $\vartheta_{1}, \ldots, \vartheta_{7}$ were chosen randomly from a uniform distribution between the maximum and minimum values allowed for these parameters. The following bounds were used for the nondimensional parameters:

$$
\begin{gathered}
0.4 \leq \vartheta_{1} \leq 1, \quad 0 \leq \vartheta_{2} \leq 1.5, \quad 0.5 \leq \vartheta_{3} \leq 2 \\
0 \leq \vartheta_{4} \leq 3, \quad 0 \leq \vartheta_{5} \leq 3, \quad 0 \leq \vartheta_{6} \leq 3, \quad 0 \leq \vartheta_{7} \leq 3
\end{gathered}
$$

The initial guesses for the initial conditions of the position and velocity states, that is, $X, Y, Z, u, v$, and $w$ are taken to be the 


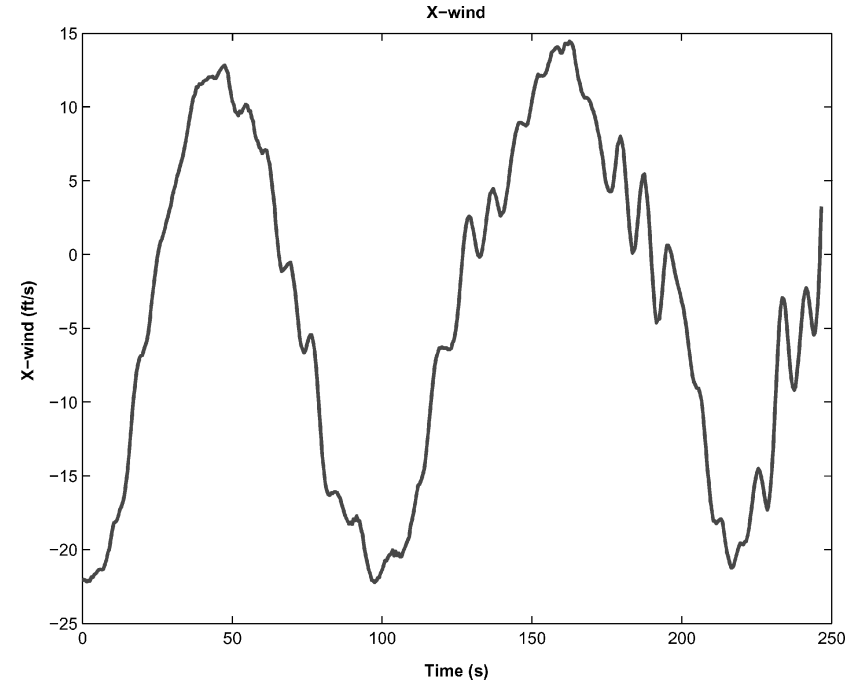

Fig. 4 Wind profile, $X$ component.

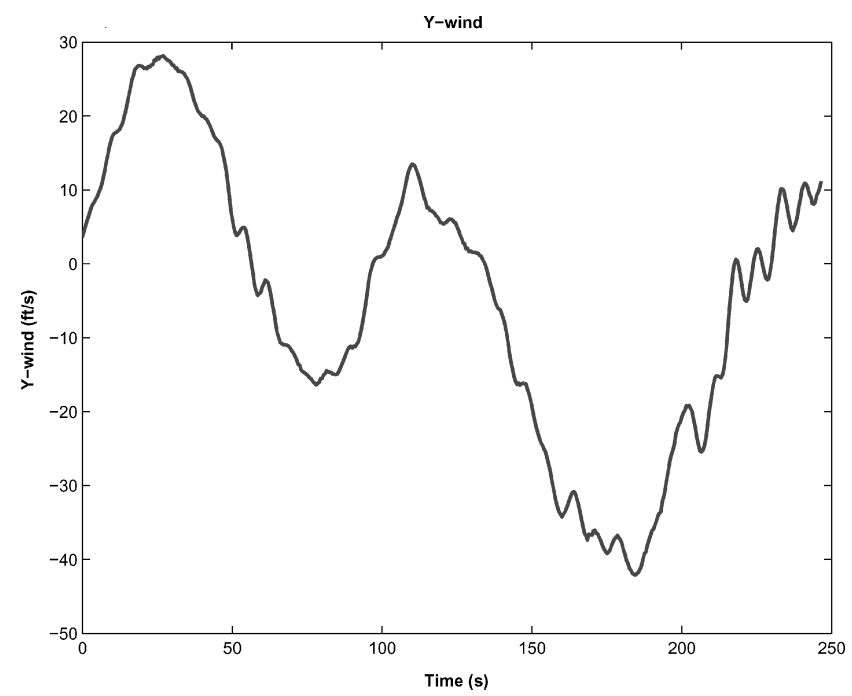

Fig. 5 Wind profile, $Y$ component.

actual measured values for those states. The other states, namely, $p$, $q, r, \phi, \theta$, and $\psi$ were chosen randomly from a uniform distribution. The following bounds were used:

$$
\begin{array}{ccc}
-5 \mathrm{deg} \leq \phi & \leq 35 \mathrm{deg}, & -20 \mathrm{deg} \leq \theta \leq 20 \mathrm{deg} \\
60 \mathrm{deg} & \leq \psi \leq 90 \mathrm{deg}, & |p| \leq 5 \mathrm{rad} / \mathrm{s} \\
|q| \leq 5 \mathrm{rad} / \mathrm{s}, & |r| \leq 5 \mathrm{rad} / \mathrm{s}
\end{array}
$$

The flight-test data used for parameter estimation are as follows. The wind input used is taken from Junge's thesis, ${ }^{3}$ which was obtained by dropping a windsonde $1 \mathrm{~h}$ prior to the G-12 test drop. The $X Y$ wind velocity components are shown in Figs. 4 and 5. Also measured were the inertial position and velocity of the parachute at the mass center of the payload (point A). These inertial variables are shown as the solid lines in Figs. 6 and 7. The inertial position and velocity are obtained from onboard global positioning system in an actual flight test.

Figure 8 shows the histogram of the prediction error as measured by the cost function $H$ in Eq. (21) before SPSA was run on each case. Note that the values of the cost function range between 9 and 490 with more than $75 \%$ of the cases being above 50 . Figure 9 shows the histogram of the prediction error as measured by the cost function $H$ in Eq. (21) after running SPSA. Over $90 \%$ of the cases attained a cost function value less than 10, and all $(100 \%)$ the cases were below 50 . Only two cases have a relatively higher cost
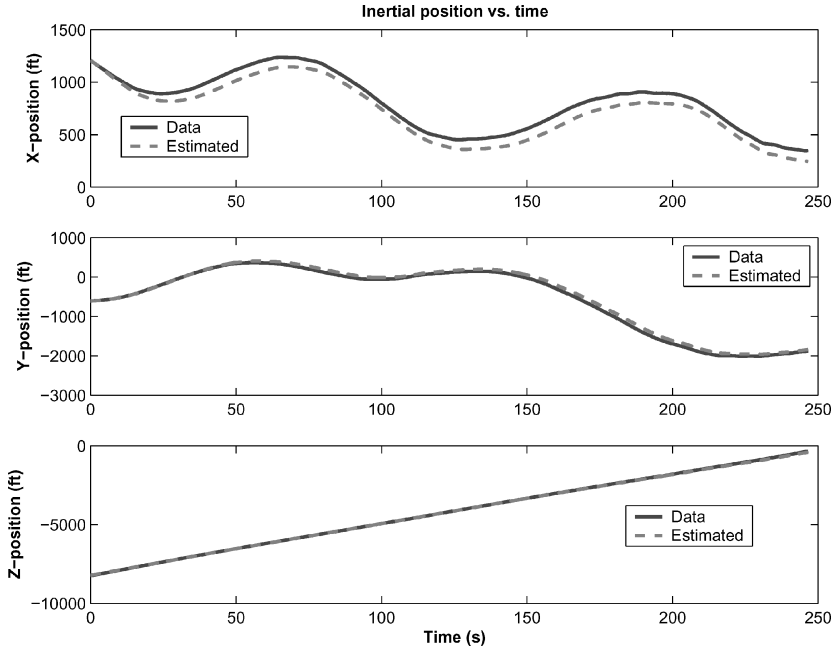

Fig. 6 Inertial position.
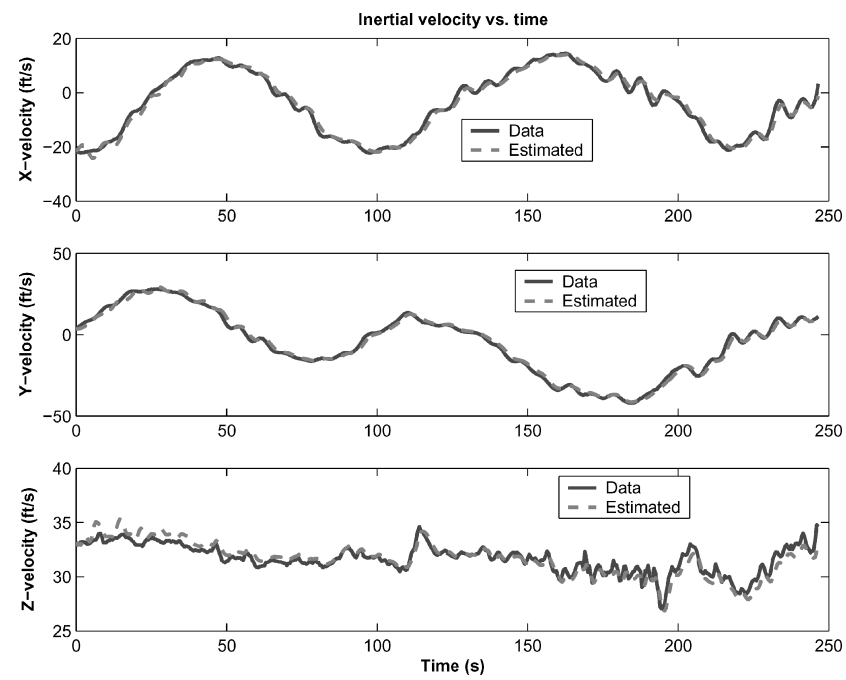

Fig. 7 Inertial velocity.

function value (above 20), indicating that for these cases SPSA did not converge to a global minimum. Those cases should be removed from further consideration as they are "outliers" that result from a local (and not global) optimization. A comparison of Figs. 8 and 9 clearly leads to the conclusion that the optimization procedure was successful. SPSA has considerably reduced both the average values of the cost function $H$ and the dispersion in cost function values. Further analysis of the statistics of the estimated parameters is warranted by these results.

Table 1 gives the results of parameter estimation for the aerodynamic and apparent mass parameters, $\vartheta_{1}, \ldots, \vartheta_{7}$. Simple statistics, computed after removing the outliers, are shown for each parameter. Also shown are the statistics of the initial guesses for these parameters, which are calculated by taking the mean, median, and standard deviation over the 40 samples generated for each parameter. The parameter estimation is successful when it reduces the initial uncertainty in the parameters; that is, when the standard deviation (STD) is reduced by the algorithm. This is the case for the $C_{d}$ offset $\vartheta_{1}$ and $C_{d}$ scale factor $\vartheta_{2}$, where the standard deviation of the SPSA estimates is smaller than that of the initial guesses. On the other hand, the standard deviation of the $C_{m}$ coefficient and the apparent mass scale factors did not decrease. This could be because the cost function did not take into account the states corresponding to the rotational motion, namely, $p, q, r, \phi, \theta$, and $\psi$ because these measurements were not available. That is, the moment coefficient scale factor and the apparent mass terms do not seem to be identifiable from the available trajectory data. 
Table 1 Results of parameter estimation

\begin{tabular}{|c|c|c|c|c|c|c|c|}
\hline \multirow[b]{2}{*}{ Parameter } & \multicolumn{3}{|c|}{ Initial guess } & \multicolumn{3}{|c|}{ Estimate (using SPSA) } & \multirow[b]{2}{*}{ Identifiable? } \\
\hline & Mean & Median & STD & Mean & Median & STD & \\
\hline$C_{d}$ offset & 0.7267 & 0.6916 & 0.1795 & 0.6249 & 0.6743 & 0.0893 & Yes (STD down 50\%) \\
\hline$C_{d}$ scale factor & 0.5718 & 0.5293 & 0.4373 & 0.0978 & 0.0270 & 0.1244 & Yes (STD down 72\%) \\
\hline$C_{m}$ scale factor & 1.2745 & 1.2542 & 0.4214 & 1.2702 & 1.2548 & 0.4234 & No \\
\hline$A_{11}$ scale factor & 1.5360 & 1.7042 & 0.7553 & 1.5047 & 1.4718 & 0.7943 & No \\
\hline$A_{15}$ scale factor & 1.5910 & 1.6353 & 0.8996 & 1.5091 & 1.4677 & 0.8690 & No \\
\hline$A_{33}$ scale factor & 1.5833 & 1.6369 & 0.6903 & 1.5871 & 1.5594 & 0.7169 & No \\
\hline$A_{55}$ scale factor & 1.6331 & 1.8531 & 0.8751 & 1.6793 & 1.7241 & 0.9011 & No \\
\hline
\end{tabular}

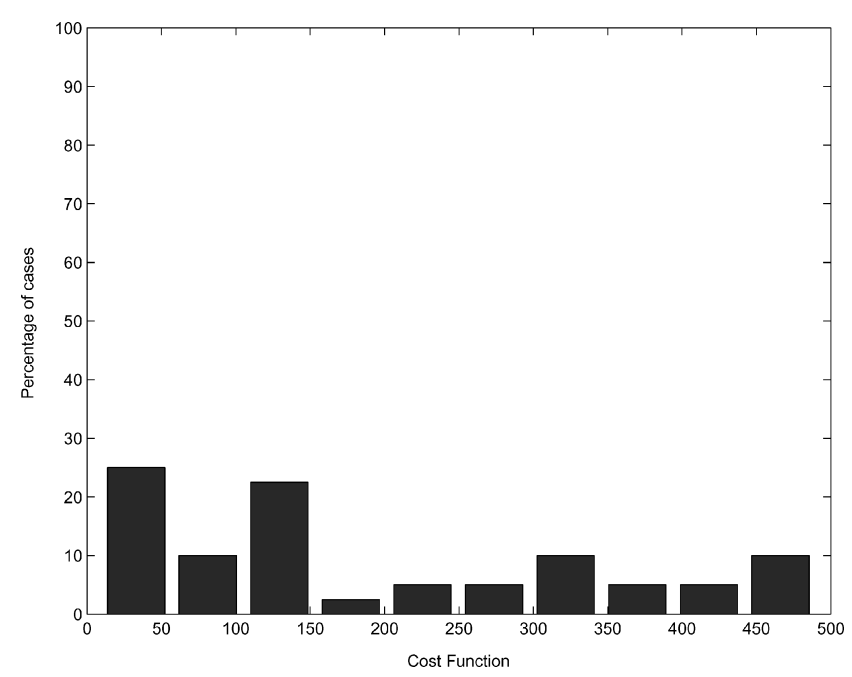

Fig. 8 Initial cost function distribution.

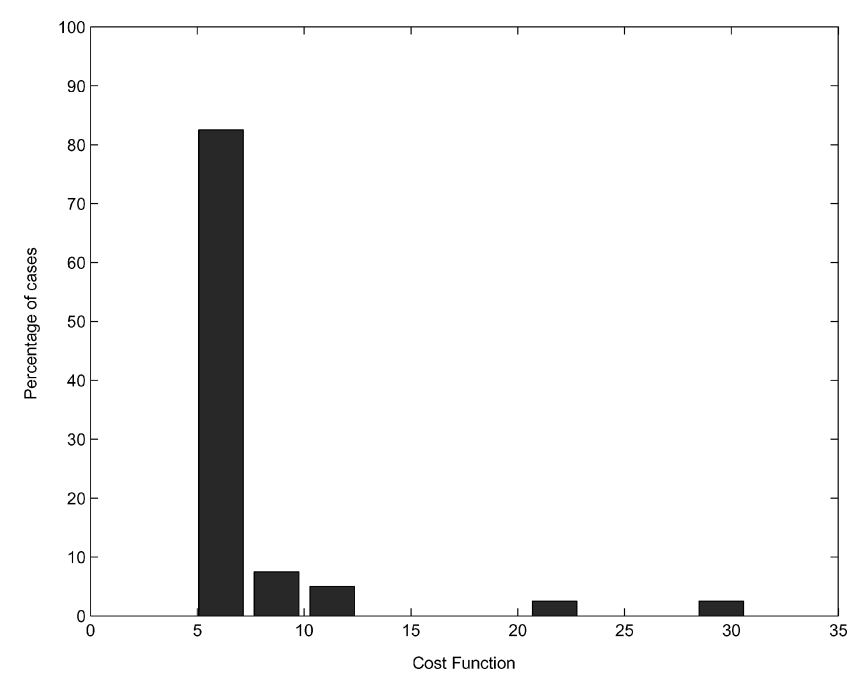

Fig. 9 Final cost function distribution.

A single model can now be constructed using the median values of the estimated parameters from Table 1. Median values after SPSA were selected for both identifiable and nonidentifiable parameters. The drag coefficient $C_{d}(\cdot)$ obtained after parameter estimation is shown in Fig. 2. Figures 6 and 7 give the measured and simulated position and velocity data of the system using the median parameter values given in Table 1 . The initial conditions used in the simulation are given in Table 2. All of the initial condition values are the median values of the estimates after running the SPSA algorithm. Figures 10 and 11 give the trajectory of the parachute on the $X-Y$ (ground) plane and in three-dimensional space, respectively. Figures 12 and 13 give the state estimation errors. The position errors fall within two parachute lengths at all times (one parachute length $=98.46 \mathrm{ft}=30 \mathrm{~m}$ ).
Table 2 Results of parameter estimation-initial conditions

\begin{tabular}{lc}
\hline \hline Intital condition & Value \\
\hline$X$ position, $\mathrm{ft}$ & 1209.0 \\
$Y$ position, ft & -606.7 \\
$Z$ position, $\mathrm{ft}$ & -8213.0 \\
$X$ velocity, $\mathrm{ft} / \mathrm{s}$ & -21.73 \\
$Y$ velocity, ft/s & 3.471 \\
$Z$ velocity, ft/s & 32.85 \\
$\phi, \mathrm{deg}$ & 18.80 \\
$\theta, \mathrm{deg}$ & -2.36 \\
$\psi, \mathrm{deg}$ & 81.76 \\
$p, \mathrm{rad} / \mathrm{s}$ & 0.139 \\
$q, \mathrm{rad} / \mathrm{s}$ & -0.024 \\
$r, \mathrm{rad} / \mathrm{s}$ & 0.110 \\
\hline \hline
\end{tabular}

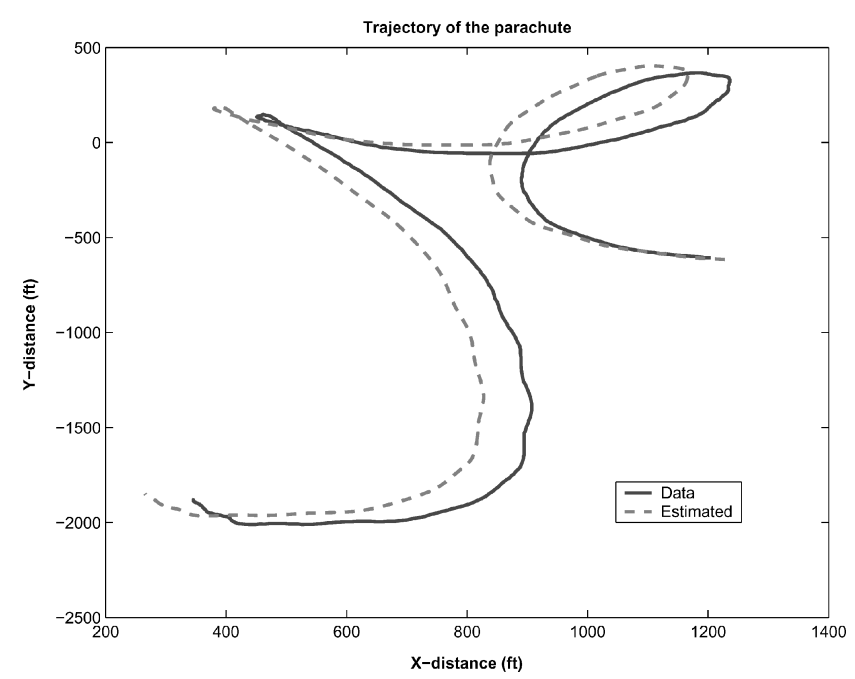

Fig. 10 Parachute trajectory in the horizontal plane.

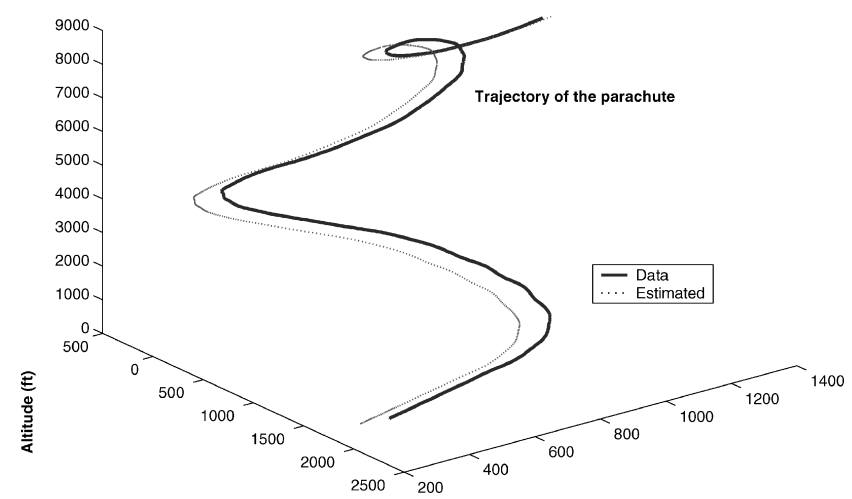

Fig. 11 Parachute trajectory. 

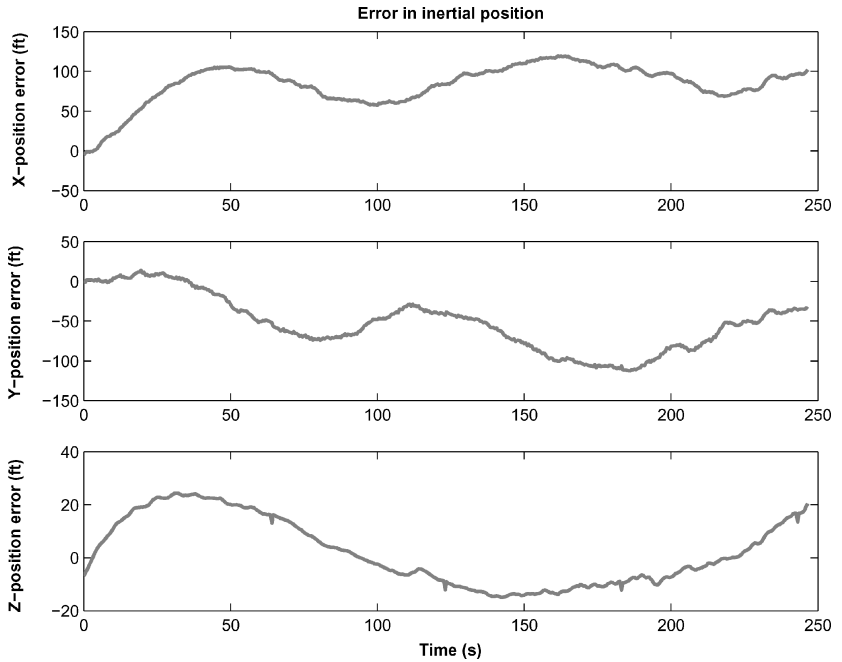

Fig. 12 Inertial position errors.
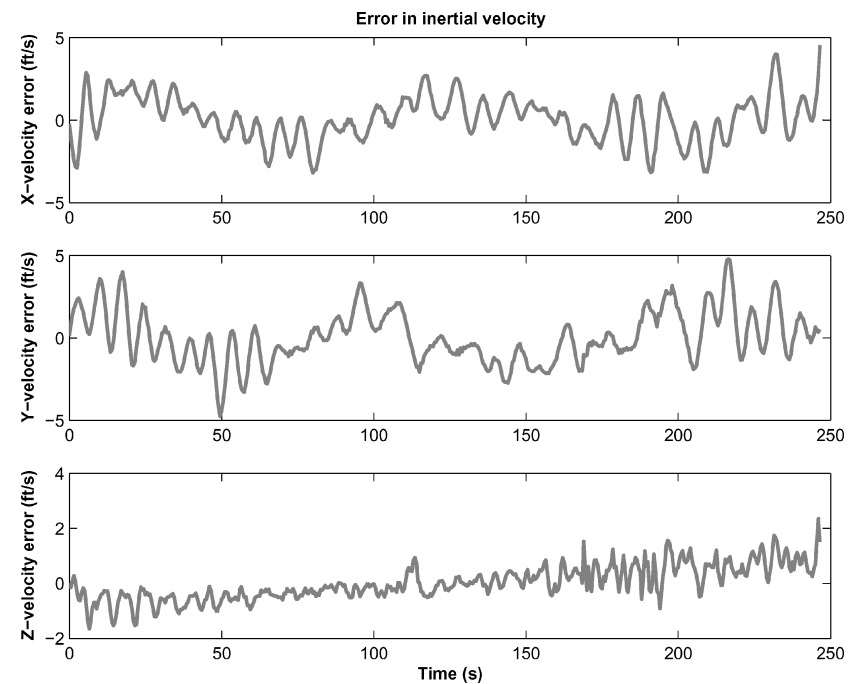

Fig. 13 Inertial velocity errors.

\section{Conclusions}

An algorithm was developed to estimate the parameters of parachute dynamic models. The algorithm is based on the constrained SPSA method, which is capable of optimizing any number of parameters in reasonable time. This is because the number of cost function evaluations needed to estimate the gradient is independent of the number of parameters to be optimized.
The algorithm was applied to the model of a G-12 parachute developed at the Naval Postgraduate School. The match between the estimated and measured position has been good, and the error was always less than two parachute lengths. However, it is clear that the parameters associated with rotational motion (e.g., $C_{m}$ ) could not be estimated. In principle, this could be because of a lack of measurements of body rates or Euler angles.

The apparent mass coefficients are also not identifiable from the available test data. From the force and moment equations one can deduce that the apparent mass coefficients would be identifiable only if the accelerations are high enough so that the effect of the apparent mass is visible in the measured states. This was not the case in this particular test.

\section{Acknowledgments}

The authors are grateful to Rick Howard, Isaac Kaminer, J. Johnson, Christopher Junge, O. Yakimenko, and V. Dobrokhodov from Naval Postgraduate School and to Scott Dellicker from the U.S. Army Yuma Proving Ground for their help with questions about parachute dynamic models and test data. This work was performed with funding from Naval Postgraduate School under Contract N00244-00-P-3258 and N00244-01-P-2540.

\section{References}

${ }^{1}$ Spall, J. C., "An Overview of the Simultaneous Perturbation Method for Efficient Optimization," John Hopkins APL Technical Digest, Vol. 19, No. 4, 1998, pp. 482-492.

${ }^{2}$ Spall, J. C., "Implementation of the Simultaneous Perturbation Algorithm for Stochasic Optimization," IEEE Transactions on Aerospace and Electronic Systems, Vol. 34, No. 3, 1998, pp. 817-823.

${ }^{3}$ Junge, C. D., "Development of a Six-Degree-of-Freedom Model for a Fully Deployed G-12 AGAS Delivery System," M.S. Thesis, Naval Postgraduate School, Monterey, CA, Dec. 2001.

${ }^{4}$ Dobrokhodov, V., Yakimenko, O., and Junge, C., "Six-Degree-ofFreedom Model of a Controllable Circular Parachute," Proceedings of AIAA Atmospheric Flight Mechanics Conference, AIAA, Reston, VA, 2002.

${ }^{5}$ Doherr, K.-F., and Saliaris, C., "On the Influence of Stochastic and Acceleration Dependent Aerodynamic Forces on the Dynamic Stability of Parachutes," Proceedings of the AIAA 7th Aerodynamic Decelerator and Balloon Technology Conference, AIAA, New York, 1981, pp. 1-9.

6“US Standard Atmosphere, 1976," U.S. Government Printing Office, Washington, D.C., 1976.

${ }^{7}$ Mosseev, Y., "Fluid Structure Interaction Simulation of the US Army G-12 Parachute," Naval Postgraduate School, Tech. Rept. 17-01-RDDOzon, Contract No. 68171-01-M-6342, Monterey, CA, Oct. 2001.

${ }^{8}$ Sadegh, P., "Constrained Optimization via Stochastic Approximation with a Simultaneous Perturbation Gradient Approximation," Automatica, Vol. 33, No. 5, 1997, pp. 889-892.

${ }^{9}$ Spall, J. C., "Multivariate Stochastic Approximation Using a Simultaneous Perturbation Gradient," IEEE Transactions on Automatic Control, Vol. 37, No. 3, 1992, pp. 332-341.

${ }^{10}$ Wang, I-J., and Spall, J. C., "A Constrained Simultaneous Perturbation Stochastic Approximation Algorithm Based on Penalty Functions," Proceedings of the American Control Conference, American Automatic Control Council, San Diego, CA, 1999, pp. 393-399. 